\title{
Range of plasma ions in cold cluster gases near the critical point
}

G. Zhang $^{1^{*}}$, H. J. Quevedo ${ }^{2}$, A. Bonasera ${ }^{1,3}$, M. Donovan², G. Dyer ${ }^{2}$, E. Gaul ${ }^{2}$, G. L. Guardo $^{3}$, M. Gulino ${ }^{3,4}$, M. La Cognata ${ }^{3}$, D. Lattuada ${ }^{3}$, S. Palmerini ${ }^{5,6}$, R. G. Pizzone ${ }^{3}$, S. Romano ${ }^{3}$, H. Smith ${ }^{2}$, O. Trippella ${ }^{5,6}$, A. Anzalone ${ }^{3}$, C. Spitaleri ${ }^{3}$, T. Ditmire ${ }^{2}$

1) Cyclotron Institute, Texas A\&M University, 77843 College Station, TX.

2) Center for High Energy Density Science, C1510, University of Texas at Austin, Austin, Texas 78712, USA

3) Laboratori Nazionali del Sud-INFN, via S. Sofia 64, 95123 Catania, Italy.

4) Libera Universita' Kore, 94100 Enna, Italy

5) Department of Physics and Geology, University of Perugia, Via A. Pascoli, 06123 Perugia, Italy

6) Istituto Nazionale di Fisica Nucleare, Section of Perugia, Via A. Pascoli, 06123 Perugia, Italy

\begin{abstract}
We measure the range of plasma ions in cold cluster gases by using the Petawatt laser at the University of Texas-Austin. The produced plasma propagated in all directions some hitting the cold cluster gas not illuminated by the laser. From the ratio of the measured ion distributions at different angles we can estimate the range of the ions in the cold cluster gas. It is much smaller than estimated using popular models, which take only into account the slowing down of charged particles in uniform matter. We discuss the ion range in systems prepared near a liquid-gas phase transition.
\end{abstract}

PACS: 52.50.Jm, 25.45._z, 36.40.Wa

Nuclear fusion from explosions of laser-heated clusters has been an active research topic for over a decade [1-14]. The explosions of cryogenically cooled deuterium $\left(D_{2}\right)$ cluster targets or near-room-temperature deuterium-methane $\left(\mathrm{CD}_{4}\right)$ cluster targets drive fusion reactions. A high intensity femtosecond laser pulse irradiated the cluster gas. This produces energetic explosions of the clusters and tens of $\mathrm{keV}$ ion plasma temperature results. DD fusion occurring within this high temperature plasma combined with beam-target fusion, between the ejected ions of the cluster and surrounding cold cluster gas, leads to a burst of fusion neutrons and protons. Following these experiments, we have modified some aspects in order to be able to measure the range of energetic ions in the cold cluster gases. Recall that the range of ions is crucial to estimate the fusion rates in the plasma. We have opportunely focused the laser in such a way that the high-energy pulse drills a "hole" in the target. We found that less than $10 \%$ of the laser energy went through the cluster gas for each shot.

A schematic view of this scenario is plotted in figure 1a, while an actual experimental result is given in figure 1b. Two Faraday cups (FC) were opportunely located: the first one (UTFC) as close as possible to the incoming laser direction $\left(-67.5^{0}\right.$ minimum) thus measuring essentially the hot plasma only; the second one (CTFC) was located at an angle around $45^{\circ}$, see fig.1, and compatible to the physical constraints of the laboratory (walls). The ratio of the FC signals gives an indication of the range of the ions in the surrounding cold cluster gas. Our experimental results show that the range of the ions in the cluster gas is almost independent of their energies and it is much

${ }^{*}$ On leave from SINAP, Shanghai, China. 
shorter than the range calculated using the popular SRIM code for instance [15]. The physics included in SRIM or similar models, is the slowing down of $\mathrm{keV}$ ions due to the interaction with electrons in the uniform gas. In our case, the gas has not an uniform density distribution but it is made of drops of different sizes, well explained by a log-normal distribution [16,17], formed during the free expansion into vacuum after the opening of the pulsed valve. Drops can be present already inside the valve before the expansion, if the gas is prepared for instance near the critical point of the liquid-gas (LG) phase transition. It is of great interest to study what happens in those cases after the gas expands. Near the second order LG phase transition, the mass distribution of the clusters follows the Fisher's law and in particular it is a power law at the critical point [18]. The free expansion might change such distribution. Theoretical calculations of a classical interacting gas, which freely expands after has been prepared near the critical point [19], do not display much variation from the predicted Fisher's cluster distribution. Thus, the cluster size distribution obtained from the expansion from the critical point might be very different respect to the lognormal distribution $[16,17]$.

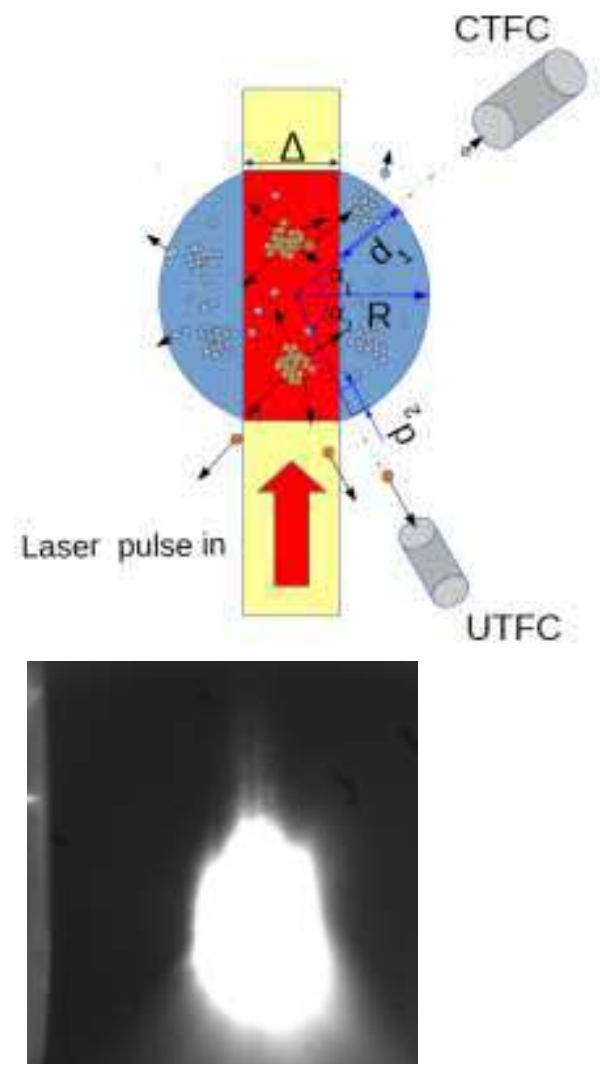

$\begin{array}{lll}\text { Figure } & 1 . & \text { a) } \\ \text { Schematic } & \text { view } & \text { of }\end{array}$ the experimental setup with the location of the two Faraday cups respect to the impinging laser beam; b) image obtained in one shot (\#9700) using a $\mathrm{D}_{2}$ gas. $\Delta$ is the laser focalization and $\mathrm{R}$ the nozzle opening. The nozzle is visible on the left hand side of fig.1b.

The laser ionizes the clusters and the ions expand quickly because of the Coulomb repulsion. Those energetic ions might move in all directions including the cold region as schematically shown in figure $1 \mathrm{a}$, and can be absorbed by a cluster on its path of size $R_{c}$ with a probability essentially given by the geometrical cross section. Notice that if no cold region is present, which can be obtained by opportunely defocusing the laser beam or decreasing (increasing) the nozzle pressure (temperature), the final ion angular distribution becomes isotropic. If the plasma ion is very energetic it can break clusters of relatively small sizes thus producing low energy ions. This 'multiplication' 
effect has been discussed already in the literature as a 'blast wave' [1-14]. Depending on the cluster size distribution we can have different ion ranges somehow dependent on the initial pressure and temperature of the gas inside the valve [20]. In this scenario we can study the influence of the initial conditions on the ion range and in particular see the effect of the system prepared in a particular state, near the LG phase transition as we will discuss below. There is no need to stress the importance of exactly measuring the range of $\mathrm{keV}$ ions in particular states of matter to understand the influence of the phase transition on nuclear fusion reactions. We also measured the fusion yields in the very same conditions and its analysis is in progress.

In our experiment, the Texas Petawatt laser (TPW) delivered 50-100 J per pulse with duration 150fs-2ps [21] to irradiate the clusters. It utilized an $\mathrm{f} / 40$ focusing mirror (10 $\mathrm{m}$ focal length) to create a large interaction volume with laser intensities sufficient to drive fusion reactions. This laser power and focusing geometry increased the neutron yield many times that of previous cluster fusion experiments [10]. Recent improvements in the laser architecture resulted in pre-pulses in the TPW system with intensities lower than $10^{-9}$ times the main pulse intensity, and did not play an important role in this experiment. The experimental setup is very similar to the one discussed in refs.[10,12] with the addition of a second FC, which was located at a distance of the order of $3 \mathrm{~m}$ for many shots. For a minor number of shots, the two FC were interchanged and located at different angles and distances in order to test any differences, due to the geometry, electronics etc. We stress that it is important to have the FCs located as far away as possible from the target to separate the very early EMP/x-ray signal from the energetic ions (around and above $100 \mathrm{keV}$ ). This was not always possible because of shielding walls surrounding the scattering chamber and the minimum distance of the FC was sometimes $0.80 \mathrm{~cm}$.

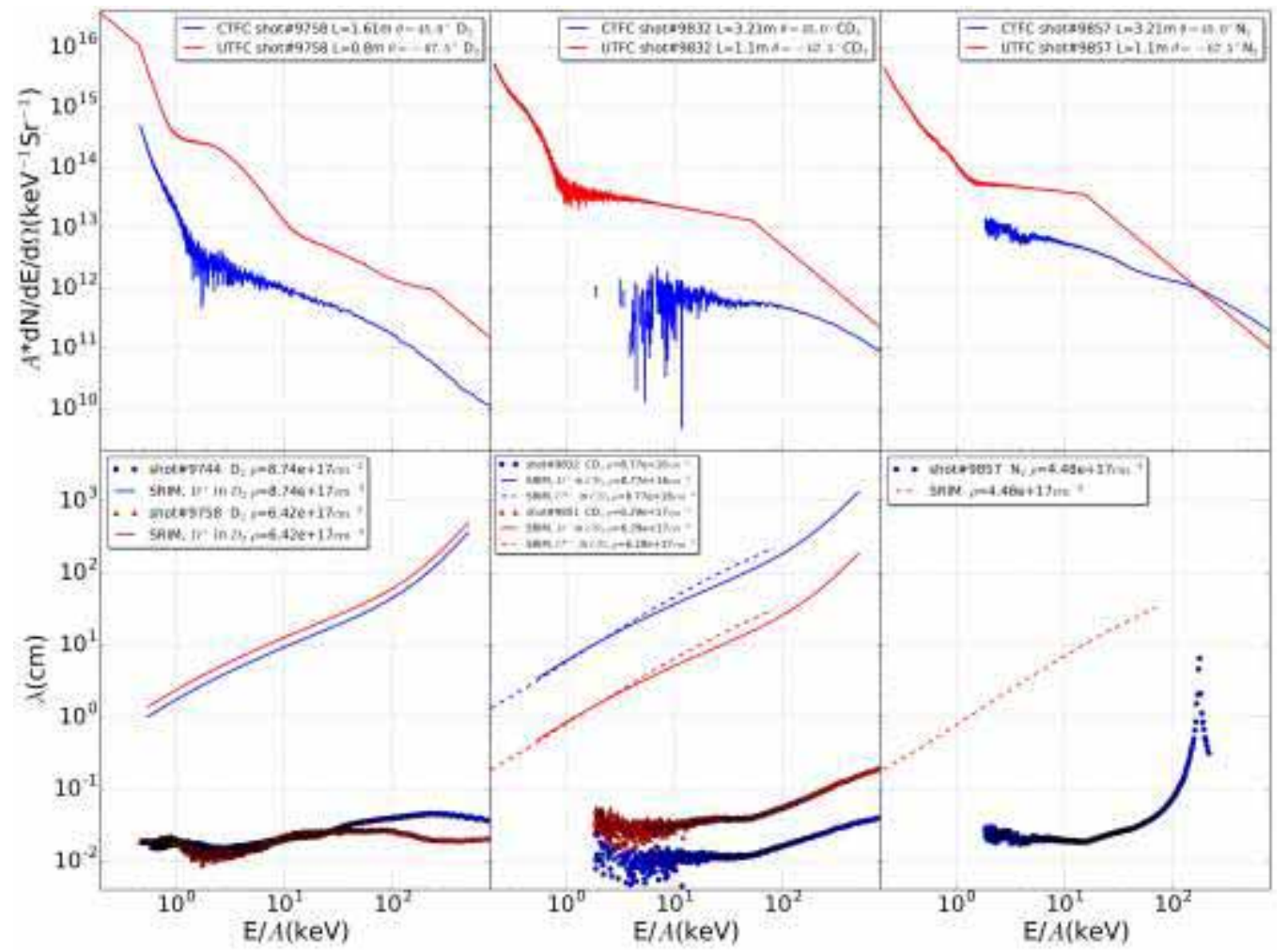

Figure 2. Top) Ion energy distributions as function of the kinetic energy per nucleon (A is the ion mass number) measured by two Faraday cups located at the angles, distances and for different gases as indicated in the figures (top panels). Bottom). Ion 
range $(\lambda)$ in the cold cluster gas obtained from equation 1 . The full and dashed lines are obtained from SRIM calculations for the different densities given in the figure [110]. The triangles and circles represent the experimental data from $D_{2}$ (left bottom panel) $\mathrm{CD}_{4}$ (center bottom panel) and $\mathrm{N}_{2}$ (right bottom panel) gases.

In figure 2, we plot the ion distributions for different shots using $\mathrm{D}_{2}, \mathrm{CD}_{4}$ and $\mathrm{N}_{2}$ gases, respectively. We have tested that the two FC measure the same ion current when $\Delta$ in figure 1 is of the order of the nozzle diameter $(5 \mathrm{~mm})$. When the plasma ions travel through cold regions of size $d_{1}, d_{2}$ in figure 1 a, they might be scattered, absorbed or slowed down. The ion distribution measured by the FC (UTFC), which sees the hot plasma, is degraded and measured by the second FC (CTFC):

$$
\frac{d^{2} N}{d E d \Omega}(C T F C)=\frac{d^{2} N}{d E d \Omega}(U T F C) e^{-\frac{\delta}{\lambda}}
$$

$\square \square \square \square \lambda$ is the ion range in the cold cluster gas, $\delta=\mathrm{d}_{1}-\mathrm{d}_{2}$. We notice that for most of our shots $\mathrm{d}_{2}$ is equal to zero.

The ion distributions and the range obtained from equation 1 are plotted in figure 2 as function of the energy per particle. The SRIM results for a homogeneous gas at different densities are given in the bottom panels with full and dashed lines. This work (see below) and previous experimental results give densities of the order of $10^{18}$ atoms $/ \mathrm{cm}^{3}$ [1-10]. Also, SRIM range results for $\mathrm{C}$ ions propagating in the cold gas are given for the $\mathrm{CD}_{4}$ case, center bottom panel of figure 2. Notice that when plotting the SRIM results as a function of the energy per nucleon $\left(2,12\right.$ and 14 for $d,{ }^{12} \mathrm{C}$ and ${ }^{14} \mathrm{~N}$ respectively), completely different stripped ions (i.e. C and D) scale quite well at the given density, (figure 2 bottom-center panel). This implies that the slowing down of ions in homogeneous matter is essentially dependent on its velocity and not on the energy. Another feature to notice is that the SRIM results depend linearly with the density, figure 2 , thus the quantity $\lambda \rho$ is expected to be independent of density, see below [15]. The SRIM calculations are in complete disagreement with our results since the experimental in-homogenous cluster gas distribution is in contrast with the theoretical uniform distribution. The EMP affects the high-energy tail of the ion distribution. Since the FCs are located at different distances the EMP dominates earlier the signal of the FC located closer. This is especially visible in the center and right panels of figure 2 since the CTFC was located at 3m while the UTFC was at $1.1 \mathrm{~m}$ from the target. The change of slope in the UTFC ion distribution below $100 \mathrm{keV} /$ nucleon reflects a sudden increase in the range. We can assume that the range is fairly constant at least in the region 1-80 keV and maybe even for higher energies. Depending on the gas density, the experimental range differs from the SRIM calculations by orders of magnitude. Notice that different clusters, either D, a mixture of $\mathrm{C}$ and $\mathrm{D}$ or pure $\mathrm{N}$ gases give similar ranges and any deviations are due to the initial pressure and temperature at the nozzle. This is consistent with the Coulomb explosion model since all ions have the same charge over mass ratio, thus we expect that a mixture of ions (for instance in $\mathrm{CD}_{4}$ ) have the same energy per nucleon distribution and similar range.

We can estimate the range of plasma ions in a cluster gas at average particle density $\rho$. The gas is composed of different clusters of size $R_{c}$. The mean free path of an ion in the cluster gas can be estimated classically as:

$$
\lambda=\frac{1}{\sigma_{c} \rho_{c}}
$$

where $\rho_{c}=\rho / N_{c}$ is the average density of clusters in the system, $N_{c}$ is the number of ions in a cluster (on average). The cross section gives the probability for an ion to collide with a cluster and it is given by: 


$$
\sigma_{c}=\pi R_{c}^{2}
$$

In the last equation we have neglected the ion dimension, which is of the order of Fermis as compared to the cluster dimension of the order of Angstroms. This has the important consequence that the probability of collision does not depend on the ions, for instance $\mathrm{D}, \mathrm{C}$ or $\mathrm{N}$, but on the cluster size. Of course heavier ions might be more effective in breaking small clusters depending on the velocity. The radius and the number of ions in the cluster are related by:

$$
R_{c}=r_{s} N_{c}^{1 / 3}=1.7 N_{c}^{1 / 3} A^{0}
$$

the inner-cluster distance among ions $r_{s}=1.7 \mathrm{~A}^{0}$ gives the density inside the cluster $\rho_{I C}=\frac{3}{4 \pi r_{s}^{3}}=4.910^{22} \mathrm{~cm}^{-3}$, i.e. of the order of solid density. This density is 3-4 orders of magnitude higher than the gas densities in figure 2. This implies that in SRIM calculations an ion travelling inside a cluster has a range 3-4 orders of magnitude shorter than the results given by the full lines in figure 2. Our experimental results are in between those two limits since the probability for an ion to collide with a cluster must be taken into account. Rearranging the equations above, we get:

$$
\lambda \rho=\frac{N_{c}^{1 / 3}}{\pi r_{s}^{2}}
$$

We have factorized out the density and obtained the probability of ion-cluster collisions dependent on the average number of ions in the cluster. As we discussed above the average number of ions in a cluster and the cluster distribution depend on the initial conditions of the gas in the nozzle, i.e. its temperature, volume and pressure [20]. We stress that our derivation is valid in the classical limit and if the gas is strongly non-uniform i.e. $\mathrm{N}_{\mathrm{c}}>>1$. This mechanism is completely different from the slowing down of ions in a uniform gas utilized in SRIM calculations where, at low kinetic energies, the range is due to ion-electron Coulomb interactions. In SRIM calculations, the quantity $\lambda \rho$ as function of the energy per particle of the ion is a universal function independent on the density of the gas.

Before we proceed further, we need to obtain the gas density from the experimental data for each shot. The plasma volume is approximately given by the 'cylindroid' of base $\Delta$ in figure 1, i.e. the laser focalization. The number of plasma ions is obtained integrating over energy the UTFC ion distribution (the 'hot' region) as plotted in figure 2 and we have estimated the EMP noise following the method discussed in [14]. For each shot we changed the pressure and temperature of the nozzle. In the top panel of figure 3 , we plot the experimental density (number of ions divided by the volume of the hot plasma) as function of the reduced pressure $\mathrm{P}_{\mathrm{r}}=\mathrm{P} / \mathrm{P}_{\mathrm{c}}$ (left panel) or reduced temperature $\mathrm{T}_{\mathrm{r}}=\mathrm{T} / \mathrm{T}_{\mathrm{c}}$ (right panel). $\mathrm{P}_{\mathrm{c}}$ and $\mathrm{T}_{\mathrm{C}}$ are the critical pressure and temperature of the liquid-gas phase transition for $\mathrm{D}_{2}(1665 \mathrm{kPa}, 38.34 \mathrm{~K}), \mathrm{CD}_{4}$ (4660kPa, 189.2K) and $\mathrm{N}_{2}(3390 \mathrm{kPa}, 126.2 \mathrm{~K})$. Notice and keep in mind that the critical pressure for $C_{4}$ and $N_{2}$ is higher that of $D_{2}$. The $D_{2}$ gas was cooled down with liquid nitrogen thus was impossible in this experiment to get closer to the critical temperature. For $\mathrm{CD}_{4}$ and $\mathrm{N}_{2}$ we used a mixed cooling system of liquid nitrogen and dry ice if needed and obtained values close to the critical point. The cluster density we measured refers to a laser-gas interaction region $4 \mathrm{~mm}$ below the nozzle and it is consistent with previous measurements using different techniques [1-10].

For the same values of $P_{r}$ and $T_{r}$ we plot in figure 3 the range $\lambda$ averaged over the 8$20 \mathrm{keV} /$ nucleon energy region, see figure 2, for the different gases, statistical errors only are plotted as well. The calculated range is at most of the order of $1 \mathrm{~mm}$ (smaller than the opening nozzle radius) and has large fluctuations near the LG phase transition, a feature that could be connected to the divergence of the correlation length 
at the critical point [22]. However, we did not succeed in this experiment to prepare the system exactly at the critical point and more systematic studies are needed in order to fully understand the influence of the LG phase transition in the expanding gas [17].

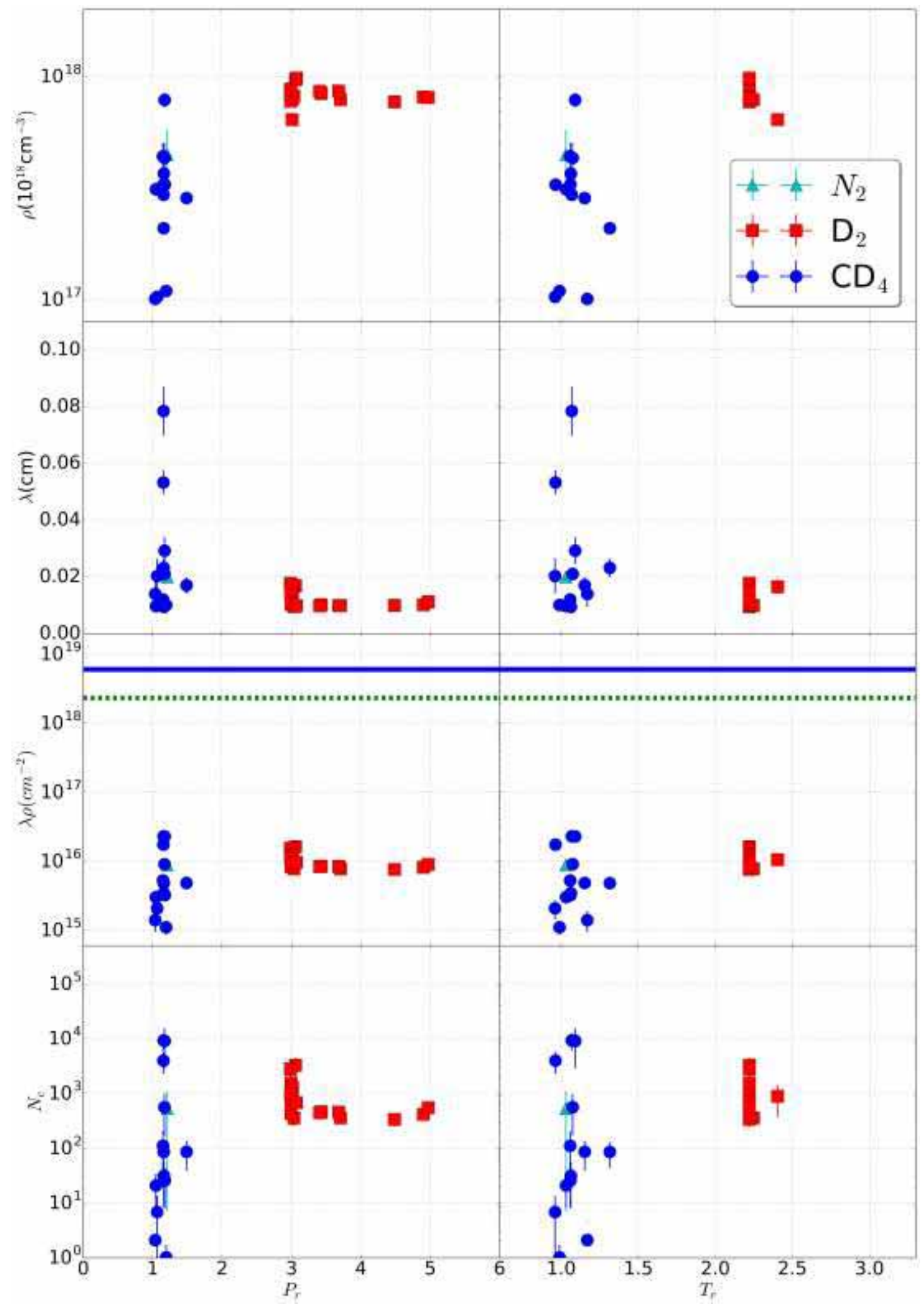


Figure 3. From top to bottom: density, range, density times range and average number of cluster obtained from eq.(5) vs reduced pressure (left panels) and reduced temperature (right panels). The full $\left(\mathrm{D}_{2}\right)$ and dashed lines $\left(\mathrm{CD}_{4}\right)$ in the third panel are the SRIM results (independent on density) averaged over the same energy range.

In figure 3 , the quantity $\lambda \rho$ is plotted as a function of $\mathrm{P}_{\mathrm{r}}$ and $\mathrm{T}_{\mathrm{r}}$. As we discussed above such quantity scales in the SRIM calculations, i.e. it does not depend on densities no matter if it is gas, liquid or solid (full and dashed lines in the figure, third panels from top). In contrast, the experimental data for a cluster gas depends on the initial pressure and temperature of the nozzle. Fluctuations for the $\mathrm{CD}_{4}$ case seem larger than the $D_{2}$ case probably because the system is initially closer to the LG critical point, see figure 3. The cluster sizes can be obtained from eq.(5) and are plotted in the bottom panels of figure 3. Notice that for the CD4 case we obtained a very low density for shots closest to the critical point. The corresponding value of $\mathrm{N}_{\mathrm{c}}$ is close to 1 which questions the validity of eq.(4) when the system is prepared near the critical region. At the critical point, the density inside the valve can be obtained from the critical compressibility $\mathrm{P}_{\mathrm{c}} /\left(\rho_{\mathrm{c}} \mathrm{T}_{\mathrm{c}}\right) \cong 0.3[19,22]$. Recall that at $\mathrm{T}_{\mathrm{c}}$ the liquid and the gas densities are the same. This relation gives critical densities a factor 2-3 smaller than the density inside the clusters $\rho_{\text {IC }}$, prepared away from the critical region [20]. This implies that the value of the $r_{s}$ quoted above might actually be larger near the critical point, thus increasing the values of $\mathrm{N}_{\mathrm{c}}$ in figure 3 , bottom panel. A quantitative estimate of this effect is not possible at this stage since the gas after being prepared in the valve expands in vacuum possibly modifying our estimates.

The Coulomb explosion model reproduces many experimental features [1-10]. The ions of an exploding cluster are subjected to an average repulsion energy given by:

$$
\frac{V_{c}}{N_{c}}=5.1 \cdot Z_{c}^{2} \cdot N_{c}{ }^{2 / 3}(\mathrm{eV})=E_{d}
$$

Where $Z_{c}$ is the average ion charge ( 1 for $D, 2$ for CD4, 7 for $N$...). Using the $N_{c}$ values given in figure 3 we get an average kinetic energy of the ions for $\mathrm{D}$ between $0.3 \mathrm{keV}$ and $11 \mathrm{keV}$ in agreement with the average kinetic energies obtained in this experiment and previous ones [1-10,14]. For CD4 and N2 gases we get a larger variation of the average kinetic energy with similar energy per particle, probably due to the initial conditions.

In conclusion, in this paper we have measured ion ranges in cold gases using the Petawatt laser at the University of Texas. We found a dependence of the ion range from the initial temperature and pressure of the gas. It seems that the range increases if the gas is prepared close to the critical point for liquid-gas phase transition. Our results are in complete contrast with SRIM calculations, which refer to homogenous gases. The method can be generalized to measure the ion range in solid or liquid secondary targets using lasers as well.

\section{Acknowledgments}

The experimental work was done at the University of Texas at Austin and was supported by NNSA Cooperative Agreement No. DE-FC52-08NA28512 and the DOE Office of Basic Energy Sciences. The analysis of the data was performed at the Texas A\&M University with support from the Cyclotron Institute (CI). Other financial and instrumental support from the Istituto Nazionale Fisica Nucleare-Italy (INFN), commissione III. G.Z. was supported by the Chinese Scholarship Council, SINAP and the CI. S.P. acknowledges the support of Fondazione Cassa di Risparmio di Perugia. One of us (AB) thanks prof. Y. G. Ma for stimulating discussion and the Chinese Academy of Science for financial support and warm hospitality during a visit 
in Shanghai.

\section{References}

[1] V. P. Krainov and M. B. Smirnov, Phys. Rep. 370, 237(2002).

[2] T. Ditmire, J. Zweiback, V. P. Yanovsky, T. E. Cowan, G. Hays, and K. B. Wharton, Nature (London) 398, 489(1999).

[3] J. Zweiback, R. Smith, T. E. Cowan, G. Hays, K. B.Wharton, V. P. Yanovsky, and T. Ditmire, Phys. Rev. Lett. 84, 2634 (2000).

[4] Y. Kishimoto, T. Masaki, and T. Tajima, Phys. Plasmas 9,589 (2002).

[5] J. Zweiback, T. E. Cowan, J. H. Hartley, R. Howell, K. B.Wharton, J. K. Crane, V. P. Yanovsky, G. Hays, R. A.Smith, and T. Ditmire, Phys. Plasmas 9, 3108(2002).

[6] I. Last and J. Jortner, Phys. Rev. Lett. 87, 033401(2001).

[7] G. Grillon et al., Phys. Rev. Lett. 89, 065005 (2002).

[8] F. Peano, R. A. Fonseca, and L. O. Silva, Phys. Rev. Lett.94, 033401 (2005).

[9] J. Davis, G. M. Petrov, and A. L. Velikovich, Phys.Plasmas 13, 064501 (2006).

[10] W. Bang, G. Dyer, H. J. Quevedo, A. C. Bernstein,E. Gaul, M. Donovan, and T. Ditmire, Phys. Rev. E 87,023106 (2013).

[11] H.Y. Lu et al., Phys. Rev. A 80, 051201 (2009).

[12] W. Bang, M. Barbui, A. Bonasera, G. Dyer, H. J. Quevedo, K.Hagel, K.

Schmidt, F. Consoli, R. De Angelis, P. Andreoli, E.Gaul, A. C. Bernstein, M.

Donovan, M. Barbarino, S. Kimura, M. Mazzocco, J. Sura, J. B. Natowitz, and T.

Ditmire, Phys. Rev.Lett. 111, 055002 (2013).

[13] M. Barbui, W. Bang, A. Bonasera, K. Hagel, K. Schmidt, J. B. Natowitz, R.

Burch, G. Giuliani, M. Barbarino, H. Zheng, G. Dyer, H. J. Quevedo, E. Gaul, A. C. Bernstein, M. Donovan, S. Kimura, M. Mazzocco, F. Consoli, R. DeAngelis, P. Andreoli,and T. Ditmire, Phys. Rev. Lett. 111, 082502 (2013).

[14] D. Lattuada, M. Barbarino, A. Bonasera, W. Bang, H. J. Quevedo, M. Warren, F. Consoli, R. De Angelis, P. Andreoli, S. Kimura, G. Dyer, A. C. Bernstein, K. Hagel, M. Barbui, K. Schmidt, E. Gaul, M. E. Donovan, J. B. Natowitz, and T. Ditmire, Phys. Rev C 93, 045808 (2016).

[15] J. F. Ziegler, M.D. Ziegler, and J. P. Biersack, Nucl.Instrum. Methods Phys. Res., Sect. B 268, 1818(2010).

[16] K. J. Mendham, N. Hay, M. B. Mason, J. W. G. Tisch and J. P. Marangos, Phys.Rev. A 64 (2001) 055201.

[17] M. Barbarino, M. Warrens, A. Bonasera, D. Lattuada, W. Bang, H. J. Quevedo, F. Consoli, R. De Angelis, P. Andreoli, S. Kimura, G. Dyer, A. C. Bernstein, K. Hagel, M. Barbui, K. Schmidt, E. Gaul, M. E. Donovan, J. B. Natowitz and T. Ditmire_Int. Jour. Mod. Phys. E25,1650063(2016).

[18] M.E. Fisher, Physics 3,255(1967).

[19] P.Finocchiaro et al., Nucl.Phys.A600,236(1996).

[20] O. F. Hagena and W. Obert, J. Chem. Phys. 56, 1793(1972).

[21] E.W. Gaul et al., Appl. Opt. 49, 1676 (2010).

[22] K.Huang, Statistical Mechanics, John Wiley \& Sons, Inc. (1987). 